\title{
E39 PARALLEL COMPUTING USING A COMMERCIAL RESERVOIR SIMULATOR
}

\author{
PAUL I. CRUMPTON, PAUL A. FJERSTAD, JOHAN BERGE \\ Schlumberger Information Solutions,
}

11 Wyndyke Furlong, Abingdon, Oxfordshire, OX14 1DZ, UK

\begin{abstract}
This paper demonstrates that simulation of models of up to 10 million cells can be performed by means of parallel simulation. This allows both geologists and reservoir engineers to include more realistic geological detail for better and more reliable production optimization.

The parallelization of the linear solver is critical to achieve calculation speed-up as more processors are used for a given model. Here the basic building block of the parallel linear solver is nested factorization. Various approaches for solving the solution matrix, in conjunction with various coloring and partitioning strategies are discussed. Several computing issues arise when applying parallel processing to complex reservoir simulation models. These issues are discussed in this paper, i.e. load balancing is problematic when using adaptive implicit (AIM) solution methods where some cells are implicit and hence more expensive than explicit cells. The choice of implicit cells is modified to achieve load balancing. Local grid refinements (LGR) are split across processors causing logistical, load-balancing and linear solver difficulties. Grid coarsening, where groups of cells are amalgamated, raises parallel computing issues. The treatment of wells, especially those that have connections, which straddle domain boundaries, will also be discussed.
\end{abstract}

\section{Introduction}

Full-field black oil or compositional simulation models with a large number of grid-blocks are in many cases a practical necessity to achieve an accurate flow description. In many reservoirs, which might have 100's or even 1000's of wells, then 100,000-200,000 grid-blocks may permit only a few grid-blocks between adjacent wells. There is therefore a continuing industry trend to include more detail in simulation models. Typically this is achieved by using a finer grid resolution.

\section{Linear Solver}

In principle the idea of parallel computing that is applied, here and many other places, is very straightforward, i.e. divide and conquer. There is nothing new in the overall parallel approach, i.e. domain decomposition, single program multiple data (SPMD) model, message passing (MPI) [5], all of which is discussed in detail elsewhere [7][3][4][8]. The linear solver used here is the popular pre-conditioned GMRES approach [6], which for reservoir simulation is totally reliant on the effectiveness of the pre-conditioner. The preconditioner used here is based on the most successful method within the reservoir simulation industry namely nested factorization [1]. So to solve the linear system, $A x=b$ which may be a block system, the matrix A is split into a series of lower and upper parts,

$8^{\text {th }}$ European Conference on the Mathematics of Oil Recovery - Freiberg, Germany, 3 - 6 September 2002 


$$
A=D+\sum_{\alpha=1}^{n l e v e l} L^{(\alpha)}+\sum_{\alpha=1}^{n l e v e l} U^{(\alpha)}
$$

where for a usual serial 3D problem nlevel $=3$ and $L^{(1)}, U^{(1)}$ represent the lower and upper connections between neighboring cells in the Z-direction. These connections in the matrix are usually the "strongest". The commonly highly stretched grids in the Z-direction usually cause this anisotropy. $L^{(2)}, U^{(2)}$ are the line-2-line connections that make up a plane of cells, and $L^{(3)}, U^{(3)}$ are plane-to-plane connections. However, in principle, the method can be applied with an arbitrary splitting of $L^{(1)}, U^{(1)}$ to $L^{(3)}, U^{(3)}$. A common looking sparsity pattern for a uniform $16 \times 16 \times 4$ grid is given in Figure 1. The unknowns are ordered such that the terms $L^{(1)}, U^{(1)}$ are closest to the diagonal, and $L^{(3)}, U^{(3)}$ are furthest away. The preconditioner $\mathrm{B}$ where $B \approx A$ is chosen as

$$
B=\left(\gamma^{(\text {nlevel })}+L^{(\text {nlevel })}\right)\left(\gamma^{(\text {nlevel })}\right)^{-1}\left(\gamma^{(\text {nlevel })}+U^{(\text {nlevel })}\right)
$$

where $\gamma$ is recursively defined by

$$
\gamma^{(\alpha)}=\left(\gamma^{(\alpha-1)}+L^{(\alpha-1)}\right)\left(\gamma^{(\alpha-1)}\right)^{-1}\left(\gamma^{(\alpha-1)}+U^{(\alpha-1)}\right), \quad \text { for } \alpha>1 .
$$

Since $B$ and $\gamma^{(\alpha)}$ are all written as product of lower and upper matrices they can easily be inverted by forward and backward substitution. The free parameter in this method is the choice of $\gamma^{(1)}$, here the matrix $\gamma^{(1)}$ is chosen to have the same dimensionality as the diagonal $\mathrm{D}$, and the matrices $\gamma^{(\alpha)}$ for $\alpha>1$ are not stored but constructed via the recursive definition in equation (4). Thus the storage overhead of constructing this factorisation is small in comparison to other methods such as incomplete LU factorization (ILU) [2]. The choice of $\gamma^{(1)}$ is crucial to the success of the method. Writing the matrix $B$ out in full gives

$$
B=\gamma^{(1)}+\sum_{\alpha=1}^{\text {nlevel }}\left(L^{(\alpha)}+U^{(\alpha)}+L^{(\alpha)}\left(\gamma^{(\alpha)}\right)^{-1} U^{(\alpha)}\right) .
$$

In order to describe this method consider $A$ to be a tri-diagonal matrix arising from solving a 1D problem i.e.

$$
A=\left(\begin{array}{ccccc}
D_{1}^{(1)} & U_{1,2}^{(1)} & & & \\
L_{2,1}^{(1)} & D_{2}^{(1)} & U_{2,3}^{(1)} & & \\
& L_{3,2}^{(1)} & D_{3}^{(1)} & U_{3,4}^{(1)} & \\
& & L_{4,3}^{(1)} & D_{4}^{(1)} & \ldots \\
& & & \ldots . & \ldots
\end{array}\right)
$$

where each entry is a block matrix, which may be of different sizes (AIM case), scalars (IMPES case), or (3x3) blocks for the fully implicit 3-phase black oil case. For this 1D problem nlevel $=1$. In this special case the factorization becomes exact as

$$
A-B=D-\gamma^{(1)}-L^{(1)}\left(\gamma^{(1)}\right)^{-1} U^{(1)}
$$

which leads to the recurrence relation

$$
\gamma_{1}^{(1)}=D_{1}, \quad \gamma_{i}^{(1)}=D_{i}-L_{i, i-1}^{(1)}\left(\gamma_{i-1}^{(1)}\right)^{-1} U_{i-1, i}^{(1)} \quad \text { for } i=2, \ldots n
$$


which is easily solved for $\gamma^{(1)}$. However, for a general sparse matrix this recurrence relation cannot be easily solved, and $\gamma^{(\alpha)}$ would not have the same non-zero entries as $D$. In order to maintain these properties a colsum or rowsum constraint is applied, so staying with the 1D example, for a general matrix

$$
\begin{array}{ll}
\qquad \gamma_{1}^{(1)}=D_{1}, \quad \gamma_{i}^{(1)}=D_{i}-\sum_{j=i}^{n} \operatorname{colsum}\left(L_{i, i-1}^{(1)}\right)\left(\gamma_{i-1}^{(1)}\right)^{-1} U_{i-1, j}^{(1)} \quad \text { for } i=2, \ldots n \\
\text { where } \quad \operatorname{colsum}\left(L_{i, i-1}^{(1)}\right)=\sum_{k=i}^{n} L_{k, i-1}^{(1)} .
\end{array}
$$

Hence $\gamma^{(1)}$ can be found without excessive storage requirements or computation. This approach is easily generalized to the nested system, where this colsum or rowsum constraint is applied to the recurrence relation at every level. With the imposition of this constraint the property $\operatorname{colsum}(A)=\operatorname{colsum}(B)$ is maintained, then within any Krylov subspace method the initial residual can be put to

$$
r=b-A y=b-A B^{-1} b,
$$

by choosing the initial approximation to $\mathrm{x}$ as $B^{-1} b$. As a consequence of the colsum property the sum of the entries of $r$ is zero [1]. This is an advantageous property for two reasons: At every iterative stage of the GMRES method the sum of the residual can be checked for being zero, this is an extremely powerful null-check that the implementation is correct. Secondly for a fully implicit case the sum of the residuals being zero can be physically interpreted as maintaining material balance throughout the linear solve. For fully implicit problems numerical evidence is overwhelming that this constraint consistently outperforms the rowsum counterpart. For the matrices arising from implicit pressure explicit saturation (IMPES) and adaptive implicit methods (AIM), the rowsum constraint is used. The recurrence relation now becomes

$$
\begin{gathered}
\gamma_{1}^{(1)}=D_{1}, \gamma_{i}^{(1)}=D_{i}-\sum_{k=2}^{i} L_{i, k-1}^{(1)}\left(\gamma_{k-1}^{(1)}\right)^{-1} \operatorname{rowsum}\left(U_{i-1, i}^{(1)}\right) \quad \text { for } i=2, \ldots n \\
\text { where } \quad \operatorname{rowsum}\left(U_{i-1, i}^{(1)}\right)=\sum_{j=i}^{n} U_{i-1, j}^{(1)}
\end{gathered}
$$

This constraint implies that the pre-conditioner is exact when the solution is a constant vector i.e.

$$
A z-A B^{-1} z=0
$$

Where $z$ denotes a vector of constant values. For IMPES matrices this means that it is possible to solve for the constant part of the pressure in one iteration. Since pressure is "smooth", we would expect that expanding pressure as a polynomial to be appropriate, and having the pre-conditioner solve for the constant part at every step is a powerful approach. However, for a fully implicit system, the saturations are not "smooth", consequently one might expect for the saturation variables this not to be such a valid approach, and indeed this is the case.

One advantage of the rowsum constraint is the ease of calculation of $\gamma^{(\alpha)}$. This can be broken up into only matrix vector multiplications of the type $\left(\gamma^{(\alpha)}\right)^{-1} x$. For a fully implicit matrix, $x$ represent the columns of rowsum $\left(U_{i-1, i}^{(1)}\right)$, so the matrix-matrix multiply in equation (12) is broken into matrix-vector multiplies, where the number depends on the size of the blocks. The 
terms $\left(\gamma^{(\alpha)}\right)^{-1} x$ are also needed for the calculation of the pre-conditioned $B^{-1}$, and consequently highly optimised code can be reused for the calculation of $\gamma^{(\alpha)}$. For the colsum constraint a matrix-matrix pre-multiply operation is required. However, this can be avoided by noting:

$$
\gamma_{\text {colsum }}^{T}=\left(\gamma_{\text {rowsum }}^{T}\right)^{T}
$$

That is to calculate $\gamma_{\text {colsum }}$ first, transpose the matrix $A^{T}$ then calculate the rowsum constraint applied to $A^{T}$ i.e. $\gamma_{\text {rowsum }}^{T}$, finally transpose back to get the colsum constraint. The disadvantage of this approach is the overhead of transposing the matrix. However the computational efficiency of the application of the rowsum constraint via matrix vector multiplies outweighs this cost.

\section{Parallel Linear solver}

For the parallel method the grid is $1^{\text {st }}$ statically partitioned into a number of domains, where each domain will be mapped onto a separate processor, see

Figure 2. Then each domain is further split into a number of colors, see Figure 4. The basic premise here is as follows. Each processor will "own" a domain and will "process" each color within the domain in parallel. Within a color there are no, or very limited number of color-2color connections. Consequently a color within a domain has no direct connections to the same color in a different domain. The connections between the colors are implemented using NNC (non-neighbour connections). These connections represent $L^{(4)}, U^{(4)}$ for the linear solver in the parallel case. The parallel execution is derived from the fact block-operations, were a block of a specific color within a domain can be performed in parallel. Equation (18) represents the sparsity of the color-2-color connections seen in Figure 4, were each entry represents the matrix from a piece of grid.

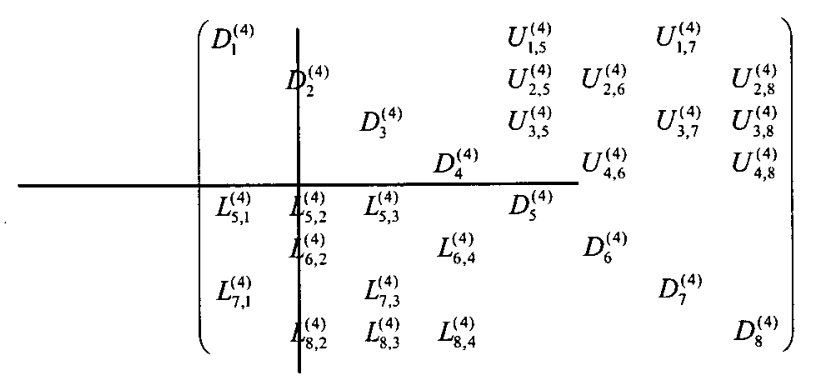

Blocks $D_{1}^{(4)}, D_{2}^{(4)}, D_{3}^{(4)}, D_{4}^{(4)}$ represent the "red" colored blocks of grid on domains 1-to-4 respectively. Similarly $D_{5}^{(4)}, D_{6}^{(4)}, D_{7}^{(4)}, D_{8}^{(4)}$ represents the "blue" colored blocks on grids 1-to-4 respectively. Since there are no connections between colors, each domain can perform operations on a color in parallel. The partition shown in equation (18) shows a $2 \times 2$ block system, it is this block system that the $4^{\text {th }}$ nesting uses in parallel. For this to be well load balanced then each domain must have the same work, and more importantly each colors within each domain must have the same work. Suppose we introduce 4 domains and 2 colors as in Figure 4 . Then the sparsity pattern of the whole system is significantly altered, in comparison with the sparsity of the original problem, Figure 1. It is worth noting that the parallel method is not the same as the serial method. Splitting the grid into domains and colors, and introducing an extra level of nesting have sacrificed numerical efficiency. For the two-color case, were no LGR's are present, the described rowsum variant is very similar to the previous work of [9][3]. 


\section{The treatment of LGR's}

One of the largest complexities to the parallel method is local grid refinements (LGR's). For a global grid with one large LGR, then it is clear that the LGR would need to be distributed across all the processors, as most of the work will be performed on the LGR. However, if we have lots of small LGR's then it would be unwise to split each small LGR across all processors, and perhaps a better option would be to let the domain that "owns" the LGR look after all the computation associated with it. However, for the case of a few medium sized LGR's the best option is less clear. Here the LGR "inherits" the partition from the global grid. If a LGR "straddles" two domains then the LGR is split across the domains. The linear solver does not know the difference between LGR planes and global planes.

One advantage of this approach is that very little user intervention is required in order to partition the grid, which has only to be done on the global grid. However, this can sometimes be restrictive and prohibit load balancing. With the approach adopted here very general problems can be solved in parallel. In Figure 5 a number of partitions are shown for a uniform base grid with increasing amounts of complexity added. Firstly the outer boundaries of the grid are coarsened, this entails removing a number of active cells from the problem, and replacing some cells with "representative cells", that are given "up-scaled" properties. Then a large LGR is added. This is distributed across all the processors evenly. Finally four smaller LGR's are added to refine around a complex well configuration. These smaller LGR's have been split along with the "base" grid. It is worth noting that the partition has been skewed to the left in order to maintain good load balancing.

\section{The treatment of AIM in parallel}

Implicit cells within an AIM method (were each matrix entry is a block) are much more expensive than explicit cells (were each matrix entry is a scalar). The choice of implicit cells is done in an effort to maintain large time steps, with a maximum $\%$ of implicitness imposed (usually around 5\% of the total number of cells). The most effective way to balance the load in parallel is to apply the AIM implicit cell choice criteria to each color within each domain. This leads to potential problems were some colors require more implicit cells to maintain a reasonable time step. Ideally a dynamic load balancing method should be applied, migrating cells throughout the run to maintain load balancing. This is an area for future research.

\section{The treatment of well}

Each well is "owned" by the domain that has the wellhead definition on it. For many cases this will not result in an evenly balanced load for the wells. If a well has connections that "straddle" domains, then extra communication is required to ensure consistency. Group control constraints are solved for on all processors, and so global communications are required after each well solve to ensure all processors have the same well information. For the reasons mentioned, the well model can become a bottleneck on large numbers of processors.

\section{Results}

The challenging model constructed here has dimensions $250 \times 400 \times 50$ or 5 million grid blocks, all cells are active. The porosity is specified to by highly heterogeneous, and permeability's are constructed via correlations. The model uses 3 phase black-oil PVT, the initial reservoir has a small gas cap, and 10 billion barrels of oil in place. The model contains over 1200 wells, 631 injector and 630 producers, these are positioned in a five-spot pattern over the entire oil region. The injectors are all under group control, injecting $90 \%$ of reservoir voidage, the producers are

$8^{\text {th }}$ European Conference on the Mathematics of Oil Recovery - Freiberg, Germany, 3 - 6 September 2002 
all under group control with a liquid rate of 300000 barrels per day. Wells with over $75 \%$ water cut are shut in.

The simulator has the new functionality to easily take sector models out of the large 5 million cell base grid. This is useful for studying the parallel performance as the large 5 or 10 million cells models cannot be run on a single CPU due to memory and time constraints. Figure 6 shows the speed-up curves for a $50 \mathrm{Kcell}, 104 \mathrm{Kcell}, 208 \mathrm{Kcell}$ and $500 \mathrm{Kcell} \mathrm{sector} \mathrm{models.} \mathrm{All} \mathrm{results}$ are from a 1-year period. Clearly for a particular number of processors the parallel efficiency increases with problem size, this is to be expected as the ratio of work/communication is increasing. These runs were performed on a 16-node Linux cluster that has a Myranet switch [10]. Figure 1 shows a plot of elapsed time against 1/proc. If the parallel execution time has a serial component (i.e. fixed time for all runs) and a parallel component (proportional to the number of processes) then this plot should be a straight line. By fitting a linear line and extrapolating to "infinite processes" gives an indication of the serial component of the run. Using this data we can estimate the $\%$ of serial time spent in the serial part of the run, that is $20 \%$ for the $50 \mathrm{~K}, 12 \%$ for the $104 \mathrm{~K}, 11 \%$ for the $204 \mathrm{~K}$ and $4 \%$ for the $500 \mathrm{~K}$. This is the encouraging trend that the fraction of time spent doing serial activity is diminishing as the problem size increases.

The 10 million cell model was constructed by inserting 2 large local grid refinements of 4 million cells each, with dimensions $200 \times 400 \times 50$. The total number of wells in the model was maintained, where the LGR's contains hundreds of wells. This huge model was run on a 72 processors $900 \mathrm{MHz}$ SUN Ultrasparc3. Similarly a run of the 5 million cell model was conducted on a 32-way $1.3 \mathrm{GHz}$ IBM P690. It was done as a proof of concept and no speed up curves are available yet.

\section{Conclusions}

It is shown that a commercial reservoir simulator can handle very large number of grid-block. The increased grid-block resolution is achieved by use of parallel processing.

Acknowledgement Dr. Gunter Roeth and Antoine Petitet at SUN Microsystems, Noble G Fortson at IBM High Performance Computing and John Wallis.

\section{References}

[1] J. R. Appleyard and I. M. Cheshire, SPE, Nested Factorisation, SPE 12264, 1983.

[2] T. F. Chan and H. A. van der Vorst, Approximate and Incomplete Factorizations, ICASE/LaRC Interdisciplinary Series in Science and Engineering, pp. 167--202. Kluwer, Dordrecht, 1997.

[3] J. Killough, A Parallel Reservoir Simulator Based On Lagal Grid Refinement, SPE 37978, 1997.

[4] J. Killough, A Scalable Parallel Reservoir Simulation on a Windows NT Based Workstation cluster., SPE 51883, 1999.

[5] MPI Consortium, The Message Passing Interface Standard, Technical report, MPI Consortium Web Pages, http://www.mcs.anl.gov/mpi/, June 1998.

[6] Y. Saad and M. H. Schultz, GMRES: A generalized minimal residual algorithm for solving nonsymmetric linear systems, SIAM J. Sci. Stat. Comput., 7 (1986), pp. 856-869 .

[7] B. Smith, P. Bjørstad and W. Gropp, Domain Decomposition: Parallel Multilevel Methods for Elliptic Partial Differential Equations, Cambridge University Press, 1996. 
[8] S. Verdiere, A. Thompson, Applications of a parallel simulator to industrial test cases, SPE 51887, 1999.

[9] J.R. Wallis et al, A new parallel iterative linear solution method for large-scale Reservoir simulation, SPOE 21209,1991 .

[10] Myrinet www.myrinet.com

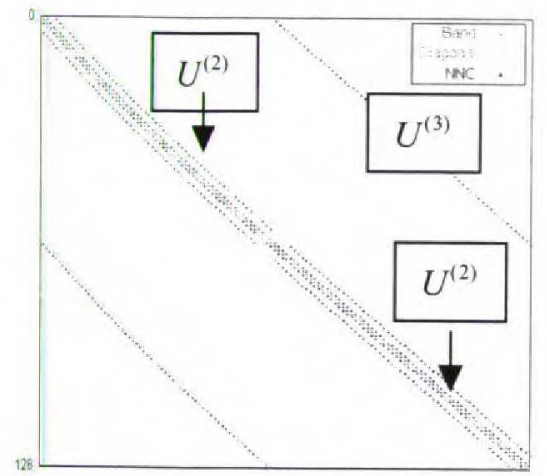

(sparsity for $1^{\text {st }} 16 \times 2 \times 4$ equations)

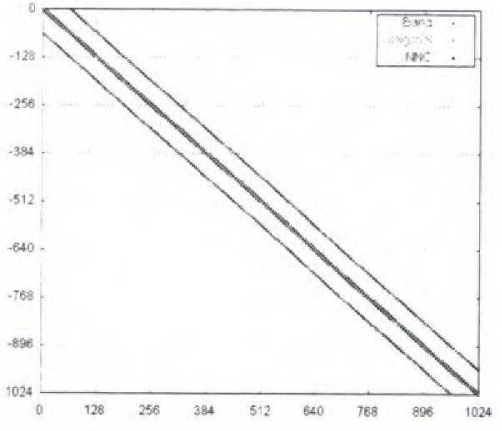

(sparsity for $16 \times 16 \times 4$ ) grid

Figure 1 Sparsity for as non-parallel uniform grid

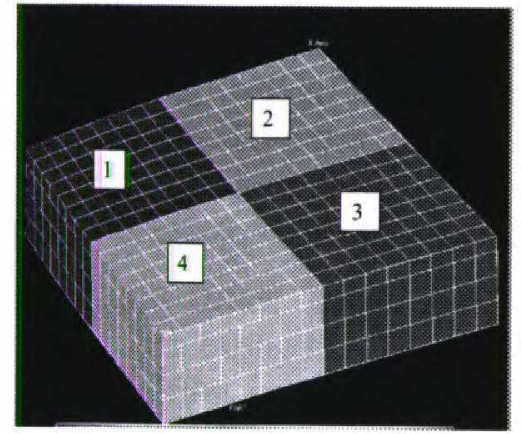

Figure 2 Four domains $16 \times 16 \times 4$ grid

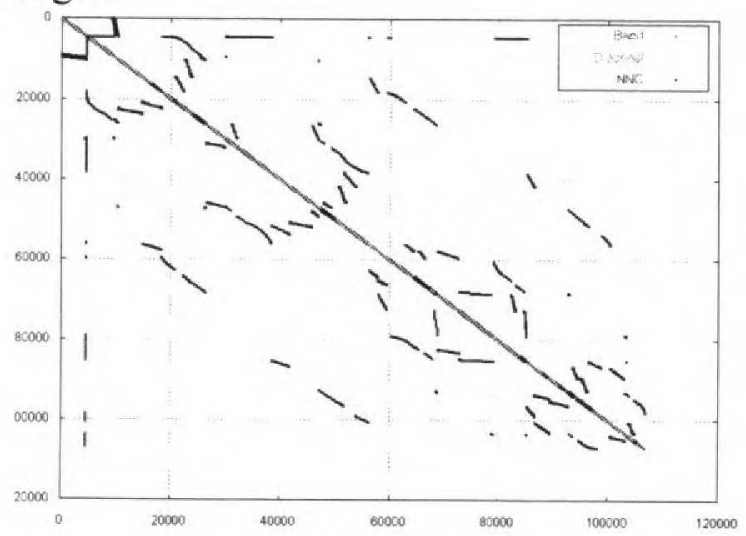

Figure 3 The sparsity from a full field model with LGR's

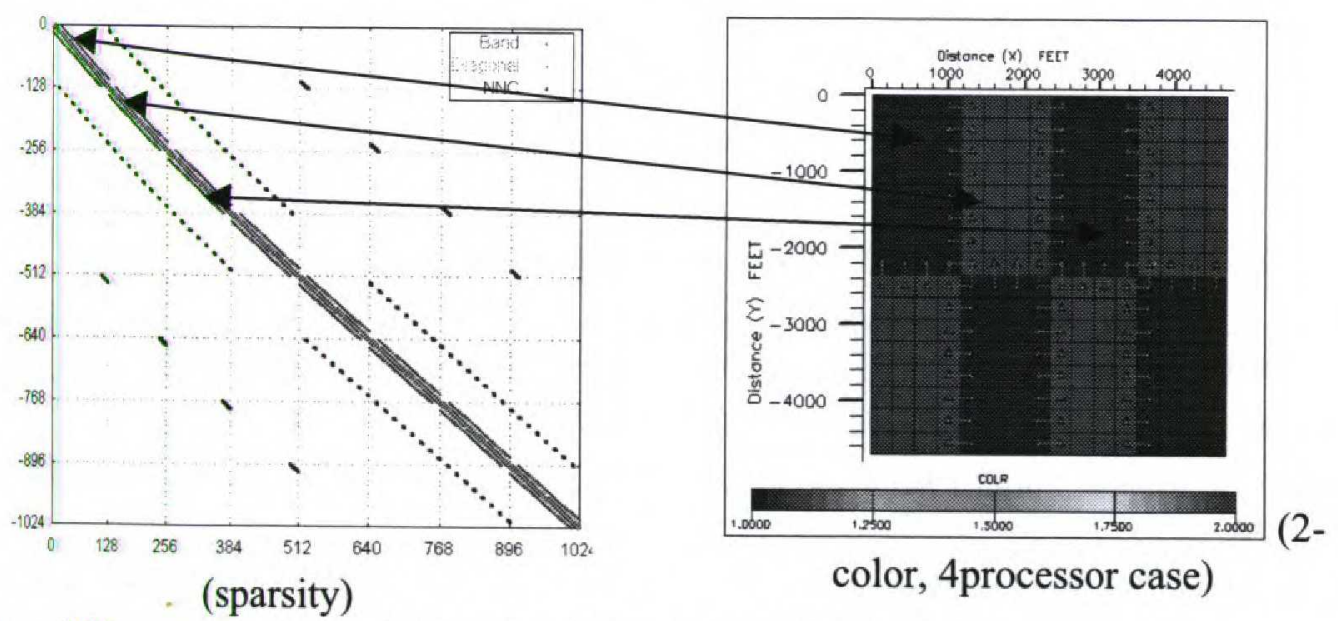

Figure 4 The sparsity and coloring for a 4-processor 2-color $16 \times 16 \times 4$ case 


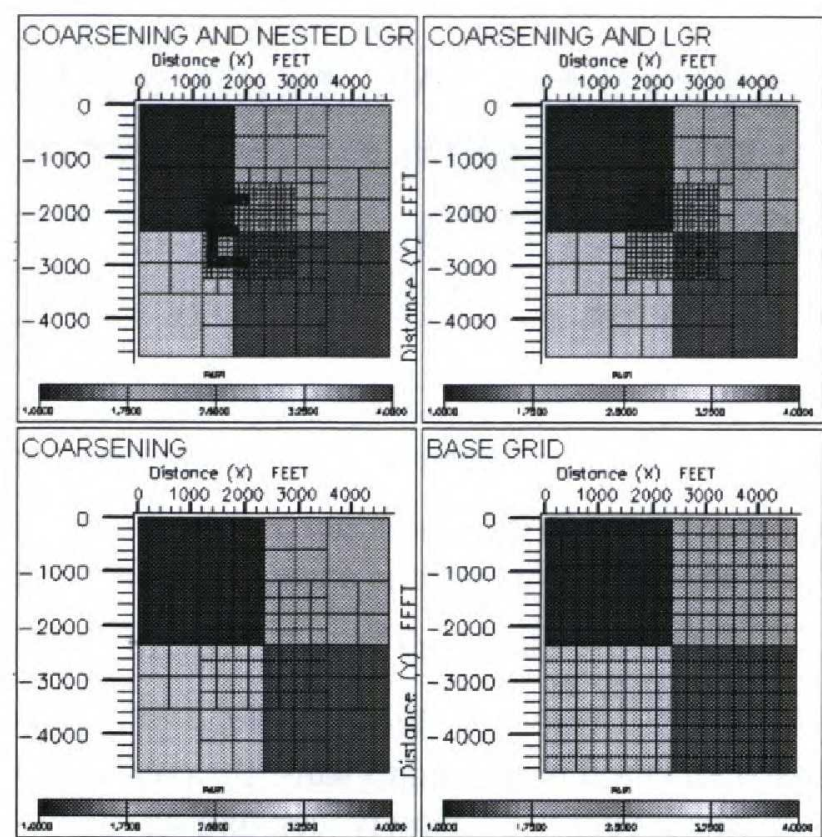

Figure 5 Partitions for 4-processor case with increasingly complex geometry

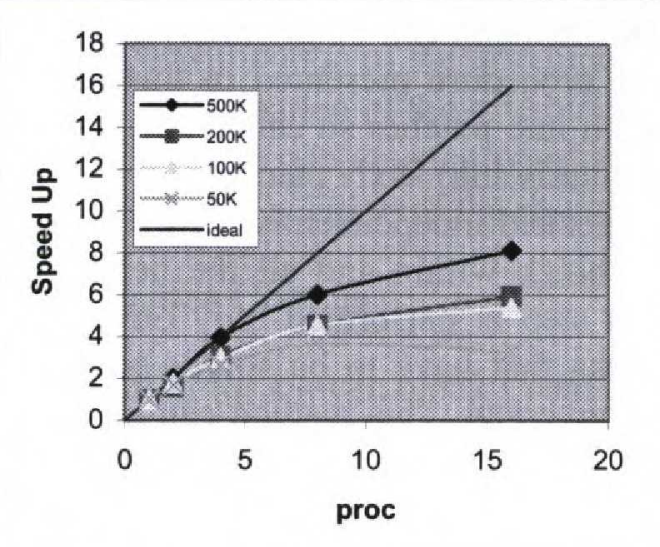

Figure 6 Speed up curves for the sector model

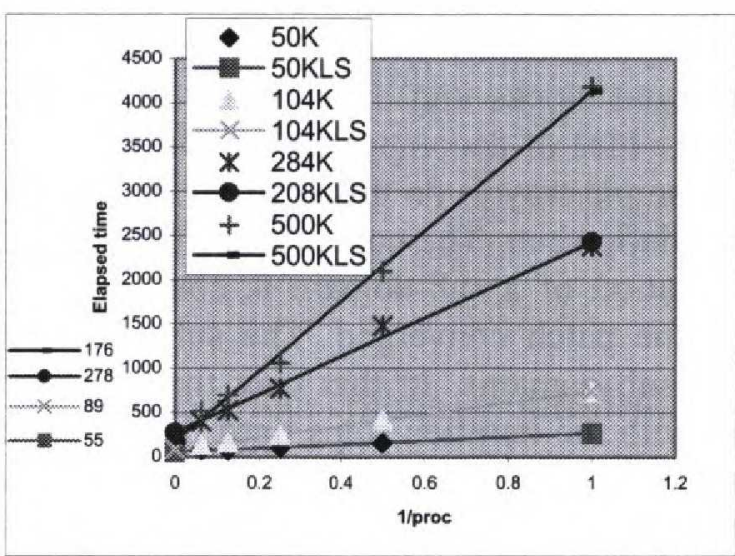

Figure 7 Elapsed time against 1/proc for the sector model. 